\title{
ON THE LENGTH OF A HILBERT ASCENDING CHAIN
}

\section{A. SEIDENBERG ${ }^{1}$}

Abstract. It is shown that if a bound $f(i)$ is placed on the degrees of the elements in some basis of an ideal $A_{i}$ in the polynomial ring $k\left[X_{1}, \cdots, X_{n}\right]$ over the field $k, i=0,1,2, \cdots$, then a bound can be placed on the length of a strictly ascending chain $A_{0}<A_{1}$ $<\cdots$. Moreover one could explicitly write down a formula for a bound $g_{n}$ in terms of $f$ and $n$.

In a polynomial ring $R=k\left[X_{1}, \cdots, X_{n}\right]$ over a field $k$ any strictly ascending chain of ideals $A_{0}<A_{1}<\ldots$ is finite, but in general no bound can be placed on the length of such an ascending chain, even if we impose on the $A_{i}$ the condition that they have a basis of monomials. It is plausible to conjecture, however, that if any sort of $a$ priori bound $f(i)$ is placed on the degrees of the elements of a basis of $A_{i}$, then the length of a chain $A_{0}<A_{1}<\cdots$ is bounded. In [2, p. 56 , Theorem 10] we proved this in the case the $A_{i}$ have monomial bases; here, we drop this condition, proving the theorem quite generally.

THEOREM. Let $f(i)$ be a nonnegative integer for $i=0,1, \cdots$ and consider ascending chains of ideals $A_{0}<A_{1}<\cdots<A_{s}$ in $k\left[X_{1}, \cdots, X_{n}\right]$, where $A_{i}$ has a basis of elements of degree $\leqq f(i)$. Then there is an integer $g_{n}$ depending only on $f$ and $n$ such that the length of any such chain is $\leqq g_{n}$. Moreover one could explicitly write down a formula for $g_{n}$ in terms of $f$ and $n$.

For the proof, we note that the theorem is trivially true for $n=0$ and obviously true for $n=1$. We make an induction on $n$.

We recall some basic results of G. Hermann [1] on computability in a polynomial ring $k\left[X_{1}, \cdots, X_{n}\right]$ (for suitable $k$ ). Given an ideal $\mathfrak{A}$ in $k[X]=k\left[X_{1}, \cdots, X_{n}\right]$ via a basis $\left(f_{1}, \cdots, f_{s}\right)$ and an $f \in k[X]$, Hermann has shown how to decide whether $f \in \mathfrak{A}$; and if it is, how to construct $g_{1}, \cdots, g_{s}$ in $k[X]$ such that $f=g_{1} f_{1}+\cdots+g_{s} f_{s}$. Given two ideals $\mathfrak{A}, \mathfrak{B}$ via bases, she has also shown how to construct $\mathfrak{A} \cap \mathfrak{B}$ and $\mathfrak{A}: \mathfrak{B}$. Moreover, she has shown how to construct an irredundant primary decomposition $\mathfrak{A}=\mathfrak{S}_{1} \cap \cdots \cap \mathfrak{S}_{t}$ of the ideal $\mathfrak{A}$. Explicit

Received by the editors October 27, 1970.

AMS 1970 subject classifications. Primary 13B25, 13E99; Secondary 13B20.

Key words and phrases. Polynomial rings, ideal chains.

${ }^{1}$ Supported in part by a National Science Foundation grant. Written in Rome, Italy, Summer 1970 . 
bounds for the number of generators, their degrees, the number of associated prime ideals, and their exponents in terms of $n$ and a bound $d$ for the $\operatorname{deg} f_{i}$ can be derived from her proofs in routine fashion (or, since the formulae can be made monotone increasing in each variable, also in terms of a bound on $n$ and $d$ ). Because of her constructivist viewpoint, Hermann assumes (in effect) that $k$ is explicitly given, i.e., that one can effectively carry out the field operations in a finite number of steps, and further (cf. [6]) that one knows explicitly how to construct a complete factorization for a given polynomial $f \in k[X]$ over $k$. Obviously, however, these bounds hold for any $k$, irrespective of any constructivist assumptions and viewpoints.

REMARK. Since the set of polynomials in $k[X]$ of degree $\leqq d$ is a finite-dimensional $k$-linear space, of a dimension given by a wellknown formula in terms of $n$ and $d$, the number $s$ of the $f_{i}$ cannot, or need not, enter into the formulae for the bounds. But from a constructivist point of view, in counting the number of steps in an algorithm, the $s$ does enter: thus we may be given superfluous $f_{i}$, and to delete these requires a number of steps depending on $s$.

Occasionally we, too, partly for the sake of simplicity in formulating our assertions, will adopt a constructivist point of view and will assume $k$ is explicitly given; but in no case do we make the assumption on factoring a polynomial.

LEMMA 1. Let $f_{i 1}, \cdots, f_{i s}, i=1, \cdots, t$, be polynomials in the ring $k\left[X_{1}, \cdots, X_{n}\right], n \geqq 0$, and $d$ an integer with $d \geqq \max \left\{\operatorname{deg} f_{i j}\right\}$. The $\left(g_{1}, \cdots, g_{s}\right)$ with $g_{i} \in k[X]$ and $f_{i 1} g_{1}+\cdots+f_{i s} g_{s}=0, i=1, \cdots, t$, form a $k[X]$-module, a finite basis of which can be computed in a number of steps depending only on $n, s, t$, and $d$ (or, also, only on a bound for these).

This is known from [1]. Here $k$ is an explicitly given field; and by a step we mean a field operation in $k$.

An $f$ in $k\left[X_{1}, \cdots, X_{n}\right]-0$ is said to be regular in $\left(X_{1}, \cdots, X_{n}\right)$ if it is of (not necessarily positive) degree $d$ in $X_{n}$ and if the coefficient of $X_{n}^{d}$ is independent of $X_{1}, \cdots, X_{n-1}$.

LEMma 2. Let $f_{1}, \cdots, f_{8}$ be polynomials in $k\left[X_{1}, \cdots, X_{n}\right], n \geqq 1$, with one of the $f_{i}$ regular in $X_{1}, \cdots, X_{n}$, and let $d$ be an integer $\geqq$ $\max \left\{\operatorname{deg} f_{i}\right\}$. Then $\left(f_{1}, \cdots, f_{8}\right) \cap k\left[X_{1}, \cdots, X_{n-1}\right]$ can be constructed in a number of steps depending only on $n, s$, and $d$ (or, also, only on a bound for these).

Proof. We are looking for the $g=g_{1} f_{1}+\cdots+g_{s} f_{s}$ with $g_{i} \in k[X]$ and with $\operatorname{deg}_{x_{n}} g=0$. Clearly we may assume $f_{s} \neq 0$ and that it is 
regular in $X_{1}, \cdots, X_{n}$. Write $g_{1}=q_{1} f_{s}+g_{1}^{\prime}$ with $q_{1}, g_{1}^{\prime} \in k[X]$ and $\operatorname{deg}_{X_{n}} g_{1}^{\prime}<\operatorname{deg}_{X_{n}} f_{0} ;$ and rewrite $g$ as $g=g_{1}^{\prime} f_{1}+\cdots+\left(g_{s}+q_{1} f_{1}\right) f_{s}$-we may assume $s \geqq 2$. Treating $g_{2}, \cdots, g_{s-1}$ similarly, we may assume that $\operatorname{deg}_{X_{n}} g_{i}<\operatorname{deg}_{X_{n}} f_{s}, i=1, \cdots, s-1$. Then $\operatorname{deg}_{X_{n}} g_{s}<2 d$ also; so we may assume $\operatorname{deg}_{X_{n}} g_{i}<2 d$ for $i=1, \cdots, s$. Writing $g_{i}=\sum g_{i j} X_{n}^{j}$ and $f_{i}=\sum f_{i j} X_{n}^{j}$, the condition $\operatorname{deg}_{x_{n}} g=0$ can be rewritten as a homogeneous linear system in the $g_{i j}$. By Lemma 1 we can compute a basis for the $\left(\cdots, g_{i j}, \cdots\right)$, and the corresponding $g$ give a basis for $\left(f_{1}, \cdots, f_{8}\right) \cap k\left[X_{1}, \cdots, X_{n-1}\right]$.

Let $A$ be an ideal in $k\left[X_{1}, \cdots, X_{n}\right]$. By the ith leading coefficient ideal $L_{i}(A)$ we mean the set of coefficients of $X_{n}^{i}$ in the polynomials $f$ in $A$ with $\operatorname{deg}_{x_{n}} f \leqq i$. The $i$ th leading coefficient ideal is contained in the $(i+1)$ th, and their union is the leading coefficient ideal $L(A)$ of $A$. By the ith subcoefficient ideal $S_{i}(A)$ we mean the set of coefficients of $X_{n}^{i}$ in the polynomials $f$ in $A$ with $\operatorname{subdeg}_{x_{n}} f \geqq i$.

Thus Lemma 2 gives $L_{0}\left(\left(f_{1}, \cdots f_{8}\right)\right)$.

Lemma 3. Let $f_{1}, \cdots, f_{s}$ be as in Lemma 2. Then for every $i$ one can construct $L_{i}\left(\left(f_{1}, \cdots, f_{s}\right)\right)$ in a number of steps depending only on $n, s$, $d$, and $i$ (or, also, only on a bound for these). One may also construct polynomials $h_{1}^{(i)}, \cdots, h_{t_{i}}^{(i)}$ of degree $\leqq i$ in $X_{n}$ whose leading coefficients will generate $L_{i}\left(\left(f_{1}, \cdots, f_{s}\right)\right)$, and one can bound the $\operatorname{deg} h_{f}^{(i)}$ in terms of $n, s, d$, and $i$ (or, also, in terms of a bound for these). Similar assertions hold for $S_{i}\left(\left(f_{1}, \cdots, f_{s}\right)\right)$, even without the regularity assumption.

The proof is like that of Lemma 2 . In the case of $S_{i}$, one has merely to consider elements of the form $g_{1} f_{1}+\cdots+g_{s} f_{8}$ with $\max \left\{\operatorname{deg} g_{j}\right\}$ $\leqq i$, so one needs no regularity assumption to depress the degrees of the $g_{j}$.

Lemma 4. Let $f_{1}, \cdots, f_{s}$ be as in Lemma 3 and let $A=\left(f_{1}, \cdots, f_{s}\right)$. Then one can construct an $X_{n}^{\rho}$ and the ideal $A: X_{n}^{\rho}$ such that $A: X_{n}^{\rho}=A$ : $X_{n}^{\rho+1}$ in a number of steps depending only on $n, s$, and $d$ (or, also, only on a bound for these). Correspondingly, one has a bound on $\rho$, on the number of elements of some basis of $A: X_{n}^{\rho}$, and on their degrees.

This is known from [1]. We merely remark that no factorizations are needed for this construction.

Lemma 5. Let $A=\left(f_{1}, \cdots, f_{8}\right)$ be as in Lemma 4. Then one can construct $L(A)$ in a finite number of steps depending only on $n, s$, and $d$ (or, also, only on a bound for these). Correspondingly one has a bound on the least $\rho$ for which $L_{\rho}(A)=L_{\rho+1}(A)=L_{\rho+2}(A)=\cdots$, on the number of elements in some basis of $L(A)$, and on their degrees. 
Proof. Let $\rho$ be such that $A: X_{n}^{\rho}=A: X_{n}^{\rho+1}$; by Lemma 4 we have such a $\rho$ at our disposal. Consider the $S_{k}(A), k \leqq \rho$, and polynomials $h_{1}^{(k)}, \cdots, h_{t_{k}}^{(k)}$ of subdegree $\geqq k$ whose coefficients of $\operatorname{deg} k$ yield a basis of $S_{k}(A)$; and let $d$ be a bound on their degrees, for $k \leqq \rho$. Then $L_{d}(A)=L(A)$. In fact, let $g \in A$ with $\operatorname{deg}_{X_{n}} g=d+e>d$. Subtracting from $g$ appropriate $k\left[X_{1}, \cdots, X_{n-1}\right]$-linear combinations of the $h_{j}^{(\mathbf{k})}$, we get a $g^{\prime}$ of the same degree in $X_{n}$ and with the same leading coefficient; so we may suppose $g \equiv 0\left(X_{n}^{\rho+1}\right)$. Then $g / X_{n}$ is also in $A$, whence $L_{d+e}(A)=L_{d+e-1}(A)$, and the lemma follows.

Thus for $A$ we have an integer $e$, depending only on $n$ and $d$-we omit the $s$ as irrelevant for present purposes-which is a bound on the least $\rho$ for which $L_{\rho}(A)=L_{\rho+1}(A)=L_{\rho+2}(A)=\cdots$ and is also a bound on the degrees of some polynomials of degree $\rho, \rho-1, \cdots$ in $X_{n}$ whose leading coefficients yield bases for $L_{\rho}(A), L_{\rho-1}(A), \cdots$ (We will then have $L_{e}(A)=L(A), e$ will be a bound on the degrees of the elements in some bases of $L_{e}(A), L_{e-1}(A), \ldots$; and there will be polynomials of degree $2 e$ whose leading coefficients yield bases for $L_{e}(A), L_{e-1}(A), \ldots$ ) We may assume $e(n, d)$ is monotone increasing in each of the variables $n, d$.

For the ideals $A_{i}$, we wish similarly to have an $e_{i}$, but do not have a regularity assumption on the $A_{i}$. To meet this we place $X_{i}^{\prime}=u_{i 1} X_{1}$ $+\cdots+u_{i n} X_{n}, i=1, \cdots, n$, where the $u_{i j}$ are $n^{2}$ indeterminates over $k$; then we work over $k(u)$ and with the transformed variables $X_{1}^{\prime}, \cdots, X_{n}^{\prime}$. We continue to have $k(u)[X] A_{i}<k(u)[X] A_{i+1}$; and $k(u)[X] A_{i}$ is regular with respect to $X_{1}^{\prime}, \cdots, X_{n}^{\prime}$. To simplify notation, we write $k$ for $k(u)$ and $X_{1}, \cdots, X_{n}$ for $X_{1}^{\prime}, \cdots, X_{n}^{\prime}$. In effect, then, we have the regularity condition for all ideals in question. Thus for $e_{i}$ we may take $e(n, f(i))$. We shall occasionally write $e_{f}$ for $e(n, f)$.

If $f$ is not already monotone increasing, we may replace it by a function $f^{\prime}$ defined as follows: $f^{\prime}(0)=f(0), f^{\prime}(i+1)=f^{\prime}(i)+f(i+1)+1$. Thus we may assume $f$ monotone increasing. We do this. Then $f_{j}(i)=f(j+i)$ is a function like $f$ for $A_{j}<A_{j+i}<\cdots$.

For inductive purposes, we generalize our theorem. Instead of just one chain $A_{0}<A_{1}<\cdots$, we will consider a finite set of (not necessarily strictly) ascending chains of ideals: $A_{0}^{(t)} \subset A_{1}^{(t)} \subset \cdots \subset A_{s}^{(t)}$, $t=1, \cdots, m$. We say that the set is strictly ascending if for each $i$, $i=0,1, \cdots, s-1$, there is at least one $t$ for which $A_{i}^{(t)}<A_{i+1}^{(t)}$. The length of such a set of chains is by definition $s+1$. Our theorem is now to be understood as asserted for any strictly ascending set of $m$ chains. The function $f$ gives a bound $f(i)$ for all the $A_{i}^{(i)}, t=1, \cdots, m$; we may assume $f$ monotone increasing. The bound $g_{n}(f)$ is to be replaced by a bound $g_{n}(m, f)$. The function $e_{f}$ continues to apply to the $A_{i}^{(t)}$ for $t=1, \cdots, m$. 
The function $f$ may be allowed to involve $n, m$ as parameters.

We remark that if $A, B$ are ideals with $A \subset B$ and $L_{i}(A)=L_{i}(B)$ for every $i$, then $A=B$.

For any integer $j$, we get an ascending chain of ideals $L_{j}\left(A_{0}^{(z)}\right)$ $\subset L_{j}\left(A_{1}^{(t)}\right) \subset \cdots \subset L_{j}\left(A_{s}^{(t)}\right)$ and thus, for $t=1, \cdots, m, m$ chains; altogether, for $j \leqq e$, we get $(e+1) m$ ascending chains (e, any integer). We have $L_{\text {ef }(0)}\left(A_{0}^{(t)}\right)=L\left(A_{0}^{(t)}\right)$ for $t=1, \cdots, m$; and consider the chains for $j \leqq e_{f}(0)$. Assume for a moment that $L_{e_{f}(0)}\left(A_{i}^{(t)}\right)=L\left(A_{i}^{(i)}\right)$ for the $s+1$ ideals $A_{i}^{(t)}$. Then clearly the $\left(e_{f}(0)+1\right) m$ chains $L_{j}\left(A_{0}^{(t)}\right) \subset \cdots \subset L_{j}\left(A_{s}^{(t)}\right), j \leqq e_{f}(0), t=1, \cdots, m$, give a strictly ascending set. By induction we have a bound $g_{n-1}\left(\left(e_{f}(0)+1\right) m, e_{f}\right)$ on $s+1$; we may assume that $g_{n-1}\left(i, e_{f}\right)$ is monotone increasing in $i$ and, inductively, that $g_{n-1}\left(i, e^{\prime}\right) \leqq g_{n-1}\left(i, e^{\prime \prime}\right)$ for any functions $e^{\prime}, e^{\prime \prime}$ such that $e^{\prime}(j) \leqq e^{\prime \prime}(j)$ for all nonnegative integers $j$. Otherwise put, we can say that if $s+1>g_{n-1}\left(\left(e_{f}(0)+1\right) m, e_{f}\right)$, then for at least one pair $(i, t)$ with $i \leqq 1+g_{n-1}\left(\left(e_{f}(0)+1\right) m, \quad e_{f}\right), \quad L_{e_{f}(0)}\left(A_{i}^{(t)}\right)<L_{e f(i)}\left(A_{i}^{(t)}\right)$; and also $L_{e f(0)}\left(A_{0}^{(t)}\right)<L_{\text {ef(i) }}\left(A_{i}^{(t)}\right)$. In this way we would get a strictly ascending set

$$
L_{e f(0)}\left(A_{0}^{(t)}\right) \subset L_{e f\left(i_{1}\right)}\left(A_{i_{1}}^{(t)}\right) \subset \cdots \subset L_{e f\left(i_{p}\right)}\left(A_{i_{p}}^{(t)}\right) ;
$$

we suppose $i_{1}, i_{2}, \cdots$ to be taken successively as small as possible. Then

$$
i_{1} \leqq 1+g_{n-1}\left(\left(e_{f}(0)+1\right) m, e_{f}\right) ;
$$

and by monotonicity of $e_{f}$, we have a bound $e_{f}\left(1+g_{n-1}\left(\left(e_{f}(0)+1\right) m, e_{f}\right)\right)$ on the degrees of the elements in some bases of the $L_{\text {ef }\left(i_{1}\right)}\left(A_{i_{1}}^{(i)}\right)$. Similarly,

$$
i_{j+1}-\left(i_{j}-1\right) \leqq 1+g_{n-1}\left(\left(e_{f}\left(i_{j}\right)+1\right) m, e_{f_{i j}}\right) .
$$

Define a function $h(j)$ as follows:

$$
h(0)=0, \quad h(j+1)=h(j)+g_{n-1}\left(\left(e_{f}(h(j))+1\right) m, e_{h^{\prime} j}\right) .
$$

Using the monotonicity properties of $g_{n-1}$, one sees by induction that $i_{j} \leqq h(j)$. Hence we have a bound $e_{f}(h(j))$ on the degrees of elements in some bases of the $L_{e f}\left(i_{j}\right)\left(A_{i_{j}}^{(t)}\right)$. Hence, too, we have the bound $g_{n-1}\left(m, e_{f}(h)\right)=1+b$ on $1+p$. Bringing the two parts of the argument together, we get

$$
h(b)+g_{n-1}\left(\left(e_{f}(h(b))+1\right) m, e_{f_{h}(b)}\right)=h(b+1)=h\left(g_{n-1}\left(m, e_{f}(h)\right)\right),
$$

which is monotone as required, as a desired bound on $s+1$. Q.E.D.

REMARK. We could make more explicit how $g_{n}(m, f)$ depends on $f$, but can do this only to a straightforward extent. It would be desirable 
to define $g_{n+1}$ in terms of $g_{n}$, where a number of parameters $m_{1}, \cdots$, $m_{k}$ may enter and $g_{n+1}\left(m_{1}, \cdots, m_{k}\right)$ will be defined in terms of $g_{n}$ at possibly a different set of values for the parameters; also other functions, recursively defined, might enter. We were able to give such a definition for the special case treated in [2].

Let $R=k\left[X_{1}, \cdots, X_{n}\right]$ and let $R \cdot m_{1}+\cdots+R \cdot m_{2}$ be a finite $R$-module; correspondingly, let $R \cdot M_{1}+\cdots+R \cdot M_{z}$ be a free $R$ module with free generators $M_{1}, \cdots, M_{z}$. Let $f_{1} M_{1}+\cdots+f_{z} M_{z}$ be an element of $R \cdot M_{1}+\cdots+R \cdot M_{2}$; by its degree we mean $\max \left\{\operatorname{deg} f_{i}\right\}$. We also call this a degree of $f_{1} m_{1}+\cdots+f_{z} m_{z}$.

CoRollary. Let $f(i)$ be a nonnegative integer for $i=0,1, \cdots$ and consider ascending chains of submodules $N_{0}<N_{1}<\cdots<N_{8}$ of the free $R$-module $R \cdot M_{1}+\cdots+R \cdot M_{z}$, where $N_{i}$ has a basis of elements of degree $\leqq f(i)$. Then there is an integer $g_{n}$ depending only on $f, n$, and $z$ such that the length of any such chain is $\leqq g_{n}$. Moreover, one could explicitly write down a formula for $g_{n}$ in terms of $f, n$, and $z$. A similar statement holds for any finite $R$-module $R \cdot m_{1}+\cdots+R \cdot m_{z}$.

Proof. We consider the case $R \cdot M_{1}+\cdots+R \cdot M_{z}$; the case $R \cdot m_{1}+\cdots R \cdot m_{z}$ follows immediately. The case $z=1$ is a reformulation of the theorem, and we make an induction on $z$. If $f_{1} M_{1}+\ldots$ $+f_{z} M_{z} \in R \cdot M_{1}+\cdots+R \cdot M_{z}$, by its leading coefficient we mean $f_{z}$. If $N$ is a submodule of $R \cdot M_{1}+\cdots+R \cdot M_{z}$, by its leading coefficient ideal we mean the ideal of leading coefficients of the elements of $N$. We obviously have $L\left(N_{0}\right) \subset L\left(N_{1}\right) \subset \cdots \subset L\left(N_{s}\right)$. Assume for a moment that $L\left(N_{0}\right)=L\left(N_{1}\right)=\cdots=L\left(N_{8}\right)$. Using $N_{0}, N_{1}$ we define a submodule $N_{1}^{\prime}$ of $R \cdot M_{1}+\cdots+R \cdot M_{z-1}$ as follows: $N_{1}^{\prime}=\left\{n_{1}-n_{0} \mid n_{0}\right.$ $\in N_{0}, n_{1} \in N_{1}$, leading coefficient of $n_{0}=$ leading coefficient of $\left.n_{1}\right\}$. Similarly, using $N_{i}$ and $N_{0}$, we define $N_{i}^{\prime}$. Clearly $N_{1}^{\prime}<N_{2}^{\prime}<\cdots<N_{s}^{\prime}$. Moreover, using the given bases of $N_{0}, \cdots, N_{s}$ we can construct (or think of constructing) bases for $N_{1}^{\prime}, \cdots, N_{s}^{\prime}$; and in this way we can put a bound on the degrees of elements in a basis of $N_{i}^{\prime}$. By induction we have a bound $b$ on $s+1$. Otherwise put, we can say that if $s+1>b$, then for at least one $i \leqq b+1, L\left(M_{0}\right)<L\left(M_{i}\right)$. By the theorem (and an induction on $z$ ), then one can compute a $B$ such that $L\left(M_{B}\right)=L\left(M_{B+1}\right)=\cdots$. Then, by another induction on $z$, we can complete the computation. The details may be omitted as being parallel to those considered in the theorem.

REMARK. The above was not written with a view to an application, though, in fact, that is how the special case of [2] arose, but one can easily envision applications. As a typical example, consider the problem of constructing the integral closure of a finite integral domain $k\left[x_{1}, \cdots, x_{n}\right]$; we think of $k[x]$ given as $k[X] / P$, with $P$ itself 
given via a basis $\left(f_{1}, \cdots, f_{s}\right)$; so some numerical data is involved. This problem has been dealt with in [5], and a variant treatment is given in [3]. In [5], the case $k(x) / k$ is separable (i.e., separably generated) was treated with the assumption that one can factor a polynomial/ $k$. In the general case, in [3], one uses some assumptions concerning $p$-independence; these are void in characteristic 0 . Thus the construction can be done in a finite number of steps for any explicitly given field of characteristic 0 ; we restrict ourselves here to this case, mainly so that there will be no doubt as to what a step is: it is a field operation in $k$. In [4] we had shown how to test whether $k[x]$ is integrally closed; and in [3] in the case $k[x]$ is not integrally closed we show how to construct an element in $k(x)$ integral over $k[x]$ but not in it. Repeating the process over and over, we get a strictly increasing chain of $k[x]$-modules contained in a finite $k[x]$-module, so the process must terminate; and one can say that one has shown how to construct the integral closure of $k[x]$. One can, however, pose the question of placing an a priori bound on the length of the chain in terms of numerical information in the data (perhaps Kronecker would have denied that a construction had been made unless this bound was supplied). This point is intended and almost explicit in [5]; it is also met in [3] via a simple computational observation of Dedekind. One can, however, also meet it by the corollary: One may assume that $x_{1}, \cdots, x_{r}$ are algebraically independent $/ k$, that $k[x]$ is integral over $k\left[x_{1}, \cdots, x_{r}\right]$, and that $x_{r+1}$ is a primitive element of $k(x) / k\left(x_{1}, \cdots, x_{r}\right)$. Let $f \in k\left[X_{1}, \cdots, X_{r+1}\right]-0$ be the irreducible polynomial satisfied by $x_{1}, \cdots, x_{r+1} / k$ and let $D\left(x_{1}, \cdots, x_{r}\right)$ be the discriminant of $f\left(x_{1}, \cdots, x_{r}, X_{r+1}\right)$. One knows that $D$ is in the conductor of $k[x]$; or, otherwise put, that the integral closure of $k[x]$ is contained in the $k\left[x_{1}, \cdots, x_{r}\right]$-module generated by $1 / D, \cdots$, $x_{r+1}^{m-1} / D$, where $m=\left[k(x): k\left(x_{1}, \cdots, x_{r}\right)\right]$. One can write the new integral element $y_{1}$ (if there is one) in the form $f_{1} / D+\cdots+f_{m-1} x_{r+1}^{m-1} / D$ with $f_{i} \in k\left[x_{1}, \cdots, x_{r}\right]$; and one can place an $a$ priori bound on the $\operatorname{deg} f_{i}$. Then one has the data for repeating this construction and can write down recursively defined bounds on the successive new integral elements $y_{1}, y_{2}, \cdots$. Applying our corollary, we get the desired bound.

ADDENDUm. We give here a free treatment of an alternative proof, suggested by the referee, for at least part of our results, avoiding, however, his use of some logical notions.

We write down the most general form for a basis of $A_{i}$, that is, we write down $r(i)$ polynomials of degree $\leqq f(i)$ with indeterminate coefficients, where $r(i)=$ the number of power-products in $X_{1}, \cdots, X_{n}$ of degree $\leqq f(i)$. The condition $A_{i}<A_{i+1}$ can then be expressed, using the first of the results cited from Hermann, in terms of polynomial 
equations and inequations over $k$ (and even over the prime field $k_{0}$ of $k$ ) in the mentioned indeterminates: more precisely, it can be written as a finite disjunction of finite conjunctions of polynomial equations and inequations. An inequation $h \neq 0$ can be rewritten as an equation $z h-1=0$ by introducing a new indeterminate $z$. Then one finds that the condition $A_{i}<A_{i+1}$ can be expressed as a conjunction of a finite set $\left\{f_{i j}=0\right\}$ of polynomial equations in indeterminates $c_{i}$. The condition holds uniformly over all field extensions of $k$ (or even of $k_{0}$ ). Hence if $\bar{c}_{i}$ is a zero in an extension field of $k$ of the set $S_{i}$ of polynomials $f_{i j}$, then in $k\left(\bar{c}_{i}\right)[X]$ we get ideals $A_{i}, A_{i+1}$ having bases of the stated kind and with $A_{i}<A_{i+1}$. By the Hilbert ascending chain theorem, the sets $S_{0}, S_{1}, \ldots$ cannot have a simultaneous solution in any extension field of $k$. Hence (by Zorn's Lemma) the polynomials in $S_{0}, S_{1}, \cdots$ generate the ideal (1) in $k\left[c_{0}, c_{1}, \cdots\right]$; hence a finite subset of the $S_{i}$ already generate (1). Hence already for a finite $s, A_{0}<A_{1}<\cdots<A_{s}$ is incompatible. In this way we get the existence of a bound $s$. Moreover, a bound can be constructed. In fact, using the elements of elimination theory (for which see, for example, our paper in Crelle J. 239/240 (1970)), we test (by computations over $k_{0}$ ) the compatibility of $A_{0}<A_{1}<\cdots<A_{8}$ successively for $s=1$, $2, \cdots$, and eventually find an $s$ for which it is incompatible. This $s$ gives a bound $g_{n}(f)$. (See, however, our previous remark.)

This argument gives $g_{n}(f)$ as general recursive in $f$. For $n=2$, following our argument, one can find a $g_{n}(f)$ primitive recursive in $f$. Even for $n \geqq 3$, where primitive recursiveness looks doubtful, we still think we have more than general recursiveness. As already noted, it would be desirable to bring to a more satisfactory expression the nature of the dependent of $g_{n}(m, f)$ on $f$.

\section{REFERENCES}

1. G. Hermann, Die Frage der endlich vielen Schritte in der Theorie der Polynomideale, Math. Ann. 95 (1926), 736-788.

2. A. Seidenberg, An elimination theory for differential algebra, Univ. Calif. Publ. Math. 3 (1956), 31-65. MR 18, 558.

3. - Construction of the integral closure of a finite integral domain, Rend. Sem. Mat. Fis. Milano (to appear).

4. - The hyperplane sections of normal varieties, Trans. Amer. Math. Soc. 69 (1950), 357-386. MR 12, 279.

5. G. Stolzenberg, Constructive normalization of an algebraic variety, Bull. Amer. Math. Soc. 74 (1968), 595-599. MR 37 \#201.

6. B. L. van der Waerden, Eine Bemerkung über die Unzerlegbarkeit von Polynomen, Math. Ann. 102 (1930), 738-739.

University of California, Berkeley, California 94720 